\title{
Research on Framework Structure for Examination Achievement Management System
}

\author{
Jun Yang
}

\begin{abstract}
Examination achievement management system is an important part of college educational administration system and plays an important role to improve the level of educational management. In view of the problem of the difficulty of software development, this article launches the research on the framework structure. First of all, this article researches basic framework structure based on Struts2, illustrates the process that Struts2 handles requests; then, this article researches 4 layer framework structure of the software and illustrates the effect of the client layer, presentation layer, business logic layer and data access layer effect; Finally, this article researches data access framework structure based on Spring JDBC, and describes the four kinds of data operation. The research results of this article can develop the examination achievement management system which adopts clear structure, easy to maintain, can also provide reference for the development of other systems.
\end{abstract}

Keywords-examination achievement; management system; framework structure; basic framework; software framework; data access framework.

\section{INTRODUCTION}

Examination is an important part of college talents training plan, which is an important means to realize the talents training target, training students' scientific research ability and creative thinking, test students' comprehensive quality and practice ability. Examination achievement management system is an essential part of education unit, essential for school policymakers and managers. Developing the examination achievement management system to make digital management of student achievement file, which can reduce working pressure of the teaching management personnel, systematically manage various services and information of educational administration, teaching, and can reduce costs, improve the work efficiency and accuracy, reasonable arrangement of time, speed up the achievement query speed, strengthen performance management, the management more standardized [1].

Framework structure is a development model of certain application based on areas, provides a wide range of software reuse, provides a unified software development kit and mode architecture for developers, stressly researches on the business logic of application, while the code of shielding the underlying implements details, so as to improve the development efficiency [2]. In order to improve the development efficiency of performance management system and safety, applicability and robustness, this article studies the framework structure, software developers can directly build

\section{School of Education and Sports, Bohai University,} Jinzhou, 121013, China

examination achievement management system, the traditional code turns to the system analysis, which reduces the technical requirements for software developers, improves the labor productivity of software development, changes the traditional software development mode.

\section{BASIC FRAMEWORK STRUCTURE}

Struts2 is the next generation products of Struts, a combined new framework on the basis of the technology of struts1 and WebWork. Struts2 takes WebWork as the core, using interceptor mechanism to deal with the user's request, make business logic controller can completely off ServletAPI [3, 4]. Struts2 has the advantages such as providing a large number of interceptors, framework based on plug-in, a variety of views support, more modular, integrated with Spring, easy to test based on the pojo, so it has been more and more widely used. It is because of the powerful features and advantages of the Struts2, which obtains a wide range of applications in software development. Struts2 basic framework structure is shown in Fig. 1.

The MVC mode is proposed by Trygve Reenskaug, a software design mode which Xerox PARC invents for programming language Smalltalk. The purpose of the MVC mode is to achieve a kind of dynamic program design, simplify the modification of subsequent to the program and expansion, and make it possible to reuse of one part of the program, through simplifying the complexity of the program, which makes the program structure more intuitive. Struts2 is an implementation of the MVC framework based on JSP Model, mainly divided into three parts of the Model (Model), view (Viewer) and controller (Controller), the design concept is decoupling performance logic and business logic through Controller, in order to improve the maintainability, extensibility and reusability of the system [5-7].

A request processing in the Struts2 framework is roughly divided into the following steps:

(1) The client submits an Http Servlet Request request, action or JSP page;

(2) The request is submitted to a series of Filter filters, Action Clean Up and Filter Dispatcher etc;

(3) Filter Dispatcher is the core of Struts2 controller, usually the last filter in the filter chain;

(4) After the request is sent to the Filter Dispatcher, Filter Dispatcher needs to call an action to deal with this Request when asks Action Mapper;

(5) If the Action Mapper decides to need to call a certain action, Filter Dispatcher puts a request to Action Proxy for processing; 
(6) Action Proxy through Configuration Manager asks framework configuration file struts. xml, find the called action class;

(7) Action Proxy creates an instance of Action Invocation, through the agent mode calls the Action;
(8) After the action is completed, return a result string, at this time in reverse order through the Intercepter blocker; Finally, an instance of Action Invocation, according to the configuration of the result element in the struts.xml, responsible for finding the corresponding result, deciding further output.

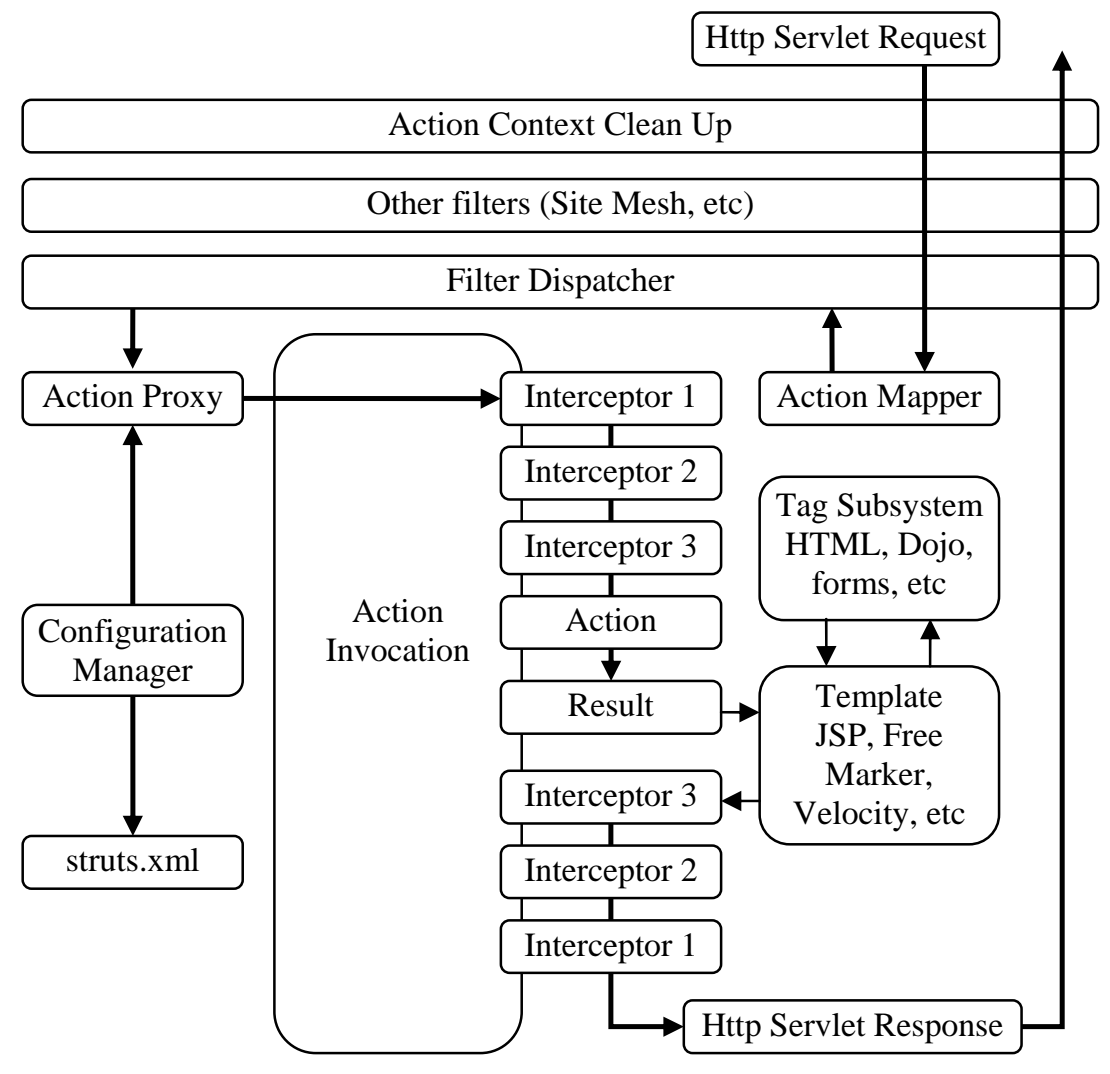

Figure 1. Struts2 framework structure

\section{SOFTWARE FRAMEWORK STRUCTURE}

B/S(Browser/Server) structure, a changed or improved structure of $\mathrm{C} / \mathrm{S}$ structure with the rise of the Internet technology. User interface is realized through WWW Browser, very few transaction logic is realized in the front(Browser), the main business logic is realized on the Server side(Server), forming the so-called three layer structure. B/S structure simplifies the client computer load, reduces the cost and the workload of system maintenance and upgrade, reducing the overall cost of users. With current technology, local area network sets up network applications of $\mathrm{B} / \mathrm{S}$ structure, and through database applications under Internet/Intranet mode, relatively easy to grasp, cost is lower. This is a one-time development in place, which can realize different people, from different locations, in different access methods(such as LAN, WAN, Internet/Intranet, etc.) to access and manipulate a common database; can effectively protect data platform and management access, server database is also very safe. Examination achievement management system uses B/S structure to develop, software framework structure is shown in Fig. 2. 


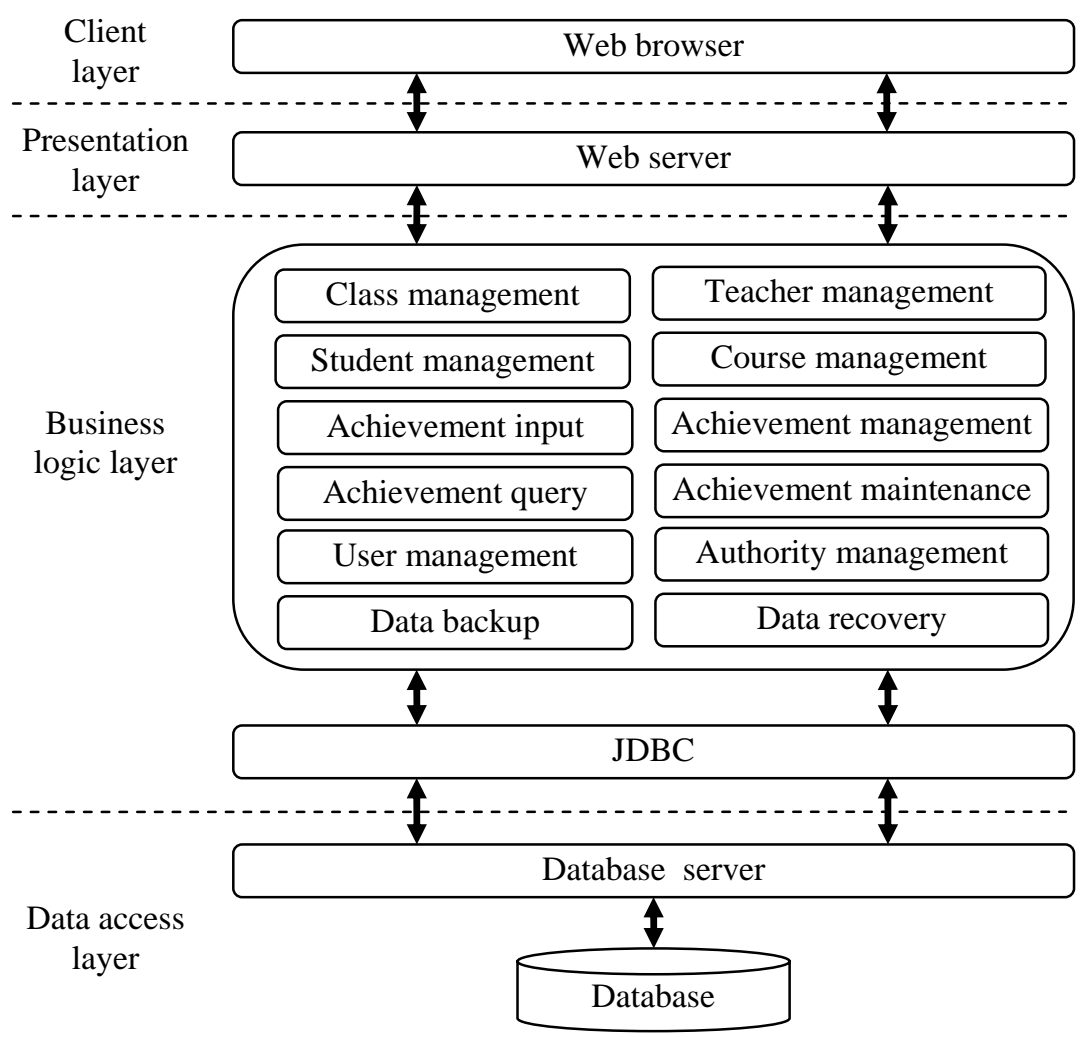

Figure 2. Software framework structure

Description of each layer in Fig. 2 shows the following [8]:

(1) Client layer (Client layer) : provides location for the clients who need to install the Web browser, users can realize their functional operations in the layer, operation users of this system include teaching management users, teachers users, students users and system administrators users.

(2) Presentation layer (Presentation layer) : provides users with the functional operations of student achievement management system, including performance management, query results, results entry, result modify, etc; also includes the administrators for system maintenance and configuration management functions.

(3) Business logic layer (Business logic layer): the position in system architecture is critical, in the middle of data access layer and presentation layer, plays a connecting role in data exchange. Focus mainly concentrated in the formulation of business rules, the relevant system design of the implementation of business processes and business requirements, etc. Also can be said that related to the system corresponding domain (Domain) logic, the business logic layer is also called the domain layer.

(4) Data access layer (Data access layer): responsible for providing data information exchange and sharing function, can realize sharing and exchange for different data formats and information. Implement Select (select),
Insert (Insert), Update (Update), Delete (Delete) operations on data table.

\section{DATA ACCESS FRAMEWORK STRUCTURE}

Spring JDBC is the Spring framework for the package of JDBC, which adopts the design pattern of template. when performs various operations on database, the original needed repetitive code which uses JDBC directly through template to complete that simplifies the use of JDBC, improves the efficiency of software development, makes the software easier to maintain and extend [9].

Spring JDBC template can undertake the work of resource management and exception handling, in order to facilitate the operation, provides three types of templates, one is the Jdbc Template, the most basic JDBC template in Spring, which uses JDBC and simple index parameter query to provide easy access to the database; two is the Named Parameter Jdbc Template, which can keep the value binding to the named parameter in the SQL when in the implementation of the query, rather than using the index parameters, three is the Simple Jdbc Template, which uses Java 5 features, such as automatic packing, general(generic) and variable parameter class to simplify the use of JDBC template. Spring JDBC framework structure is shown in Fig. 3.

As can be seen from the Fig. 3, Spring JDBC operations on the data are divided into four categories, one is the basic data manipulation, the conventional data operation, specific operation method can be that insert or 
update data, query data, call a stored procedure, etc.; two is the $\mathrm{BLOB} / \mathrm{CLOB}$ type data operations, including insert data and read data; three is the auto increment primary key, including in the sequence way and the table way to generate the value of primary keys; four is the OO way to access to the database, that is in an object-oriented way to access to the database.

\section{CONCLUSIONS}

With the development of computer technology, especially the development of network technology and database technology, people's life and work style have changed greatly. Network technology makes it possible that the communication between computers, information sharing, and database technology provides the functions such as data storage, information retrieval, information analysis, so as to make the work more efficiently. The overall task of examination achievement management system is to achieve achievement management which is systematic, scientific, standardized and automatic, the main task is to use a computer to carry out integrated management on test scores [10], including query, modify, add, delete, analysis, statistics, reports, and other functions, also including system maintenance, data backup, data recovery and other functions. Framework normally makes a certain abstract for the application, which uses some common functions needed to make software modules, developing software just needs to write the code for a specific position. This article is researches on framework structure specifically for the achievement management, not only significantly development efficiency is improved, and the developed software has the advantages such as clear structure, stable performance, easy maintenance and easy to transplant and so on, which has important practical significance for the development of examination achievement management system, at the same time, also can provide reference for the development of other systems.

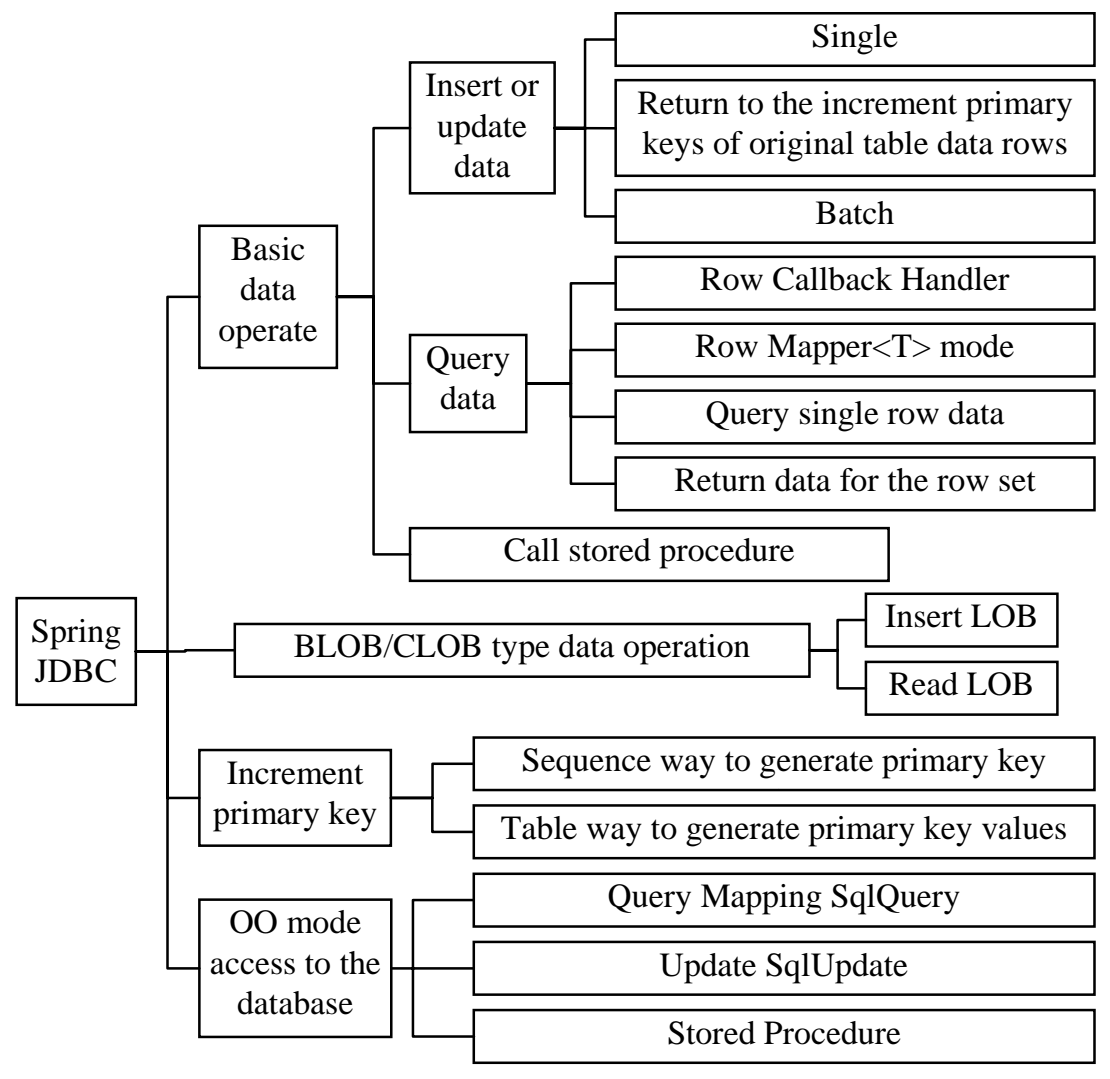

Figure 3 Spring JDBC framework structure

\section{ACKNOWLEDGEMENTS}

The research work was supported by "12th Five Year Plan" of educational science planning project of Liaoning province No. JG13CB030, 2014 annual general undergraduate higher education reform project of Liaoning province (Research on action primary education professional training mode reform based on Standard),
2013 annual Bohai University teaching reform project No. JG13YB004.

\section{REFERENCES}

[1] J. J. Sha, X. Xu. The Demand Analysis and Design of Score Management System in College. Value Engineering, 32(19), pp. 204-205, 2013.

[2] Y. C. Ren, D. Y. Jinag, T. X. Xing, et al. Research on software 
development platform based on SSH framework structure. Procedia Engineering, 15(2), pp. 3078-3082, 2011.

[3] Z. G. Zhang. Development and application on Struts2 framework technology. Computer CD Software and Applications, 16(23), pp. 119-120, 2013.

[4] Baidu Baike. Struts2. http :// baike.baidu.com/view/1566725.htm? from_id $=10711345 \&$ type $=$ syn $\& \quad$ fromtitle $=$ Struts $2 \&$ fr $=$ aladdin, 2014-11-21.

[5] Y. B. Guo, S. M. Lu. Universal design based on Struts2 framework. Computer Programming Skills \& Maintenance, 21(11), pp. 21-23, 2014.

[6] Red and black Union (yapian8's Special column). Struts2 Detailed framework. http : // www.2cto.com / kf/201408/ 329840.html, 2015-1-20.
[7] J. Li, Y. J. Chen, X. B. He. The Design and Implementation of Performance Management System Based on Struts2 Architecture. Journal of China West Normal University(Natural Sciences), 35(3), pp. 281-285, 2014.

[8] F. Wang. The Design and Implementation on Student Achievement Management System. Master's Degree of University of Electronic Science and Technology, 2013.

[9] L. Zhang, W. X. Zhang. Application of Vegetables Transportation and Sales Integrated Management System in Date Persistent Based on Spring and JDBC. Journal of Anhui Agricultural Sciences, 40(7), pp. 4401-4403, 2012.

[10] C. Q. Li. The The design of student achievement management system based on SSH2. Computer Knowledge and Technology, 10(21), pp. 4955-4958, 2014. 